\title{
Symmetry constraints for the emission angle dependence of Hanbury-Brown-Twiss radii
}

\author{
U. Heinz, ${ }^{1,2}$ A. Hummel, ${ }^{1}$ M. A. Lisa, ${ }^{1}$ and U. A. Wiedemann ${ }^{3}$ \\ ${ }^{1}$ Physics Department, The Ohio State University, Columbus, Ohio 43210 \\ ${ }^{2}$ Kavli Institute for Theoretical Physics, University of California, Santa Barbara, California 93106-4030 \\ ${ }^{3}$ Theoretical Physics Division, CERN, CH-1211 Geneva 23, Switzerland
}

(Received 29 June 2002; published 9 October 2002)

\begin{abstract}
We discuss symmetry constraints on the azimuthal oscillations of two-particle correlation (Hanbury-BrownTwiss interferometry) radii for noncentral collisions between equal spherical nuclei. We also propose a method for correcting in a model-independent way the emission angle dependent correlation function for finite eventplane resolution and angular binning effects.

DOI: 10.1103/PhysRevC.66.044903

PACS number(s): 25.75.Ld, 24.10.Nz, 24.85.+p
\end{abstract}

\section{INTRODUCTION}

In the study of heavy-ion collisions two-particle BoseEinstein correlations are an important tool for extracting information on the space-time structure of the collision zone at freezeout [1]. At nonzero impact parameter the reaction zone formed by the two overlapping nuclei is initially spatially deformed. When viewed along the beam direction $z$, it is longer in the direction $y$ perpendicular to the reaction plane (defined by the beam axis and the impact parameter vector pointing in $x$ direction) than in the reaction plane. The reaction plane orientation can be determined event-by-event from anisotropies in the collective flow of the emitted particles [2-4]: at lower collision energies one exploits the directed flow of the protons near projectile and target rapidity ("bounceoff") [5], while at high energies (where the directed flow becomes very weak) one uses the elliptic flow of the produced particles at midrapidity [6].

Having extracted the orientation of the reaction plane from the final distribution of the emitted particle momenta, one can then address the question of their spatial distribution relative to the reaction plane by measuring $[7,8]$ two-particle correlations as a function of the azimuthal emission angle $\Phi$ (i.e., the direction of the transverse momentum vector $\boldsymbol{K}_{\perp}$ of the emitted particle pairs relative to the impact parameter $\boldsymbol{b}$ ) [9-11]. Complementing the spectral information on the momentum-space structure of the source with space-time information from the correlation functions severely constrains models for the dynamical evolution of the reaction zone [1]. For noncentral collisions interesting questions that can be addressed in this way are the origin and manifestation of anisotropic collective flow and its consequences for the space-time evolution of the fireball, from which information about the intensity of rescattering effects and the degree of thermalization in particular during the early stages of the collision can be extracted [see, e.g., Ref. [12]].

For quantum statistical correlations between, say, identical pions the measured two-pion correlation function $C(\boldsymbol{q}, \boldsymbol{K})$ is related to the emission function (single-pion phase-space distribution at freezeout) $S(x, \boldsymbol{K})$ by [1]

$$
C(\boldsymbol{q}, \boldsymbol{K})=1+\left|\left\langle e^{i \boldsymbol{q}(\mathbf{x}-\boldsymbol{\beta} t)}\right\rangle(\boldsymbol{K})\right|^{2} .
$$

Here $\boldsymbol{q}=\boldsymbol{p}_{1}-\boldsymbol{p}_{2}$ and $\boldsymbol{K}=\frac{1}{2}\left(\mathbf{p}_{1}+\boldsymbol{p}_{2}\right)$ are the relative and aver- age momentum of the pion pair, respectively, $\boldsymbol{\beta}=\left(\boldsymbol{p}_{1}\right.$ $\left.+\boldsymbol{p}_{2}\right) /\left(E_{1}+E_{2}\right)$ is the velocity of the pair, and the average $\langle\cdots\rangle$ is taken with the emission function:

$$
\langle f(x)\rangle(\boldsymbol{K})=\frac{\int d^{4} x f(x) S(x, \boldsymbol{K})}{\int d^{4} x S(x, \boldsymbol{K})} .
$$

If the space-time structure of $S(x, \boldsymbol{K})$ can be approximated by a Gaussian, the resulting correlator $C(\boldsymbol{q}, \boldsymbol{K})$ is again a Gaussian in the relative momentum $\boldsymbol{q}$ and can be fully characterized by six Haubury-Brown-Twiss (HBT) radius parameters $R_{i j}^{2}$ which are functions of the pair momentum $\boldsymbol{K}$ [1]:

$$
C(\boldsymbol{q}, \boldsymbol{K})=1+\exp \left[-\sum_{i, j=o, s, l} q_{i} q_{j} R_{i j}^{2}(\boldsymbol{K})\right] .
$$

Here $\boldsymbol{q}$ is conventionally decomposed into orthogonal components along the beam direction $(l=$ longitudinal $)$, parallel to the transverse pair momentum $\boldsymbol{K}_{\perp}(o=o u t)$ and along the remaining third direction $(s=s i d e)$. In this $(o s l)$ frame the pair velocity has components $\boldsymbol{\beta}=\left(\beta_{\perp}, 0, \beta_{l}\right)$. The radius parameters $R_{i j}^{2}$ are then calculable from the spatial correlation tensor

$$
S_{\mu \nu}(\boldsymbol{K})=\left\langle x_{\mu} x_{\nu}\right\rangle(\boldsymbol{K})-\left\langle x_{\mu}\right\rangle(\boldsymbol{K})\left\langle x_{\nu}\right\rangle(\boldsymbol{K}) \equiv\left\langle\tilde{x}_{\mu} \tilde{x}_{\nu}\right\rangle,
$$

$(\mu, \nu=0,1,2,3)$, which describes, for pairs with momentum $\boldsymbol{K}$, the widths in space-time of the emission function $S(x, \boldsymbol{K})$ around the point of highest emissivity [1]. The spatial correlation tensor is specified in coordinates $x_{\mu}$ attached to the reaction plane: $x_{3}=z$ is the beam direction, $x_{1}=x$ is the direction of the impact parameter $\boldsymbol{b}$, and $x_{2}=y$ points perpendicular to the reaction plane. For the spatial correlation tensor this choice of coordinates is natural since the reaction plane is a symmetry plane for the collision. The relations between $R_{i j}^{2}$ and $S_{\mu \nu}$ are $[1,10]$

$$
R_{s}^{2}=\frac{1}{2}\left(S_{11}+S_{22}\right)-\frac{1}{2}\left(S_{11}-S_{22}\right) \cos (2 \Phi)-S_{12} \sin (2 \Phi),
$$




$$
\begin{aligned}
R_{o}^{2}= & \frac{1}{2}\left(S_{11}+S_{22}\right)+\frac{1}{2}\left(S_{11}-S_{22}\right) \cos (2 \Phi)+S_{12} \sin (2 \Phi) \\
& -2 \beta_{\perp}\left(S_{01} \cos \Phi+S_{02} \sin \Phi\right)+\beta_{\perp}^{2} S_{00} \\
R_{o s}^{2}= & S_{12} \cos (2 \Phi)-\frac{1}{2}\left(S_{11}-S_{22}\right) \sin (2 \Phi) \\
& +\beta_{\perp}\left(S_{01} \sin \Phi-S_{02} \cos \Phi\right) \\
R_{l}^{2}= & S_{33}-2 \beta_{l} S_{03}+\beta_{l}^{2} S_{00} \\
R_{o l}^{2}= & \left(S_{13}-\beta_{l} S_{01}\right) \cos \Phi+\left(S_{23}-\beta_{l} S_{02}\right) \sin \Phi \\
& -\beta_{\perp} S_{03}+\beta_{l} \beta_{\perp} S_{00} \\
R_{s l}^{2}= & \left(S_{23}-\beta_{l} S_{02}\right) \cos \Phi-\left(S_{13}-\beta_{l} S_{01}\right) \sin \Phi
\end{aligned}
$$

The radius parameters $R_{i j}^{2}$ are functions of the pair rapidity $Y=\frac{1}{2} \ln \left[\left(1+\beta_{l}\right) /\left(1-\beta_{l}\right)\right]$, the magnitude $K_{\perp}$ of the transverse pair momentum, and its angle $\Phi$ relative to the reaction plane (the azimuthal emission angle). In Eqs. (5) we only indicated the explicit $\Phi$ dependence arising from the azimuthal rotation of the (osl) system relative to the reactionplane-fixed $(x y z)$ system:

$$
x_{o}=x \cos \Phi+y \sin \Phi, \quad x_{s}=-x \sin \Phi+y \cos \Phi .
$$

In addition to this explicit $\Phi$ dependence, there is an implicit one [10] arising from the dependence of the emission function $S(x, \boldsymbol{K})=S\left(x, y, z, t ; Y, K_{\perp}, \Phi\right)$ on the emission angle $\Phi$; this generates a $\Phi$ dependence of the components of the spatial correlation tensor $S_{\mu \nu}$. In this note we work out the symmetry constraints on the $\Phi$ dependence of $S_{\mu \nu}$ and study their implications on the $\Phi$ dependence of the HBT radius parameters after the explicit $\Phi$ dependence shown in Eqs. (5) is folded in.

\section{SYMMETRIES OF THE EMISSION FUNCTION AND SPATIAL CORRELATION TENSOR}

For spherical colliding nuclei the emission function is symmetric under reflection at the reaction plane:

$$
\text { I: } S\left(x, y, z, t ; Y, K_{\perp}, \Phi\right)=S\left(x,-y, z, t ; Y, K_{\perp},-\Phi\right) \text {. }
$$

This leads to the following symmetry relations for the spatial correlation tensor:

$$
\text { I: } S_{\mu \nu}\left(Y, K_{\perp}, \Phi\right)=\theta_{1} S_{\mu \nu}\left(Y, K_{\perp},-\Phi\right),
$$

with

$$
\theta_{1}=(-1)^{\delta_{\mu 2}}+\delta_{\nu 2}
$$

Thus, symmetry I relates the components of $S_{\mu \nu}$ at emission angle $\Phi$ with those at angle $-\Phi$ at the same pair rapidity $Y$ and transverse momentum $K_{\perp} . S_{02}, S_{12}$, and $S_{23}$ are odd under this symmetry $\left(\theta_{1}=-1\right)$, all other components are even $\left(\theta_{1}=+1\right)$.
If the two nuclei have equal mass, the emission function is also symmetric under interchange of projectile and target. In the center of mass system centered at the collision point, this translates into a point reflection symmetry at the origin:

$$
\text { II: } S\left(x, y, z, t ; Y, K_{\perp}, \Phi\right)=S\left(-x,-y,-z, t ;-Y, K_{\perp}, \Phi+\pi\right) \text {. }
$$

For the spatial correlation tensor this implies

$$
\text { II: } S_{\mu \nu}\left(Y, K_{\perp}, \Phi\right)=\theta_{2} S_{\mu \nu}\left(-Y, K_{\perp}, \Phi+\pi\right),
$$

with

$$
\theta_{2}=(-1)^{\delta_{\mu 0}}+\delta_{\nu 0}
$$

Symmetry II relates $S_{\mu \nu}$ at emission angle $\Phi$ for forwardgoing pairs $(Y>0)$ with $S_{\mu \nu}$ at emission angle $\Phi+\pi$ for backward-going pairs $(Y<0)$, and vice versa. For midrapidity pairs $(Y=0)$ it relates the spatial correlation tensor at emission angles $\Phi$ and $\Phi+\pi$, providing a useful second constraint on the emission angle dependence. $S_{01}, S_{02}$, and $S_{03}$ are odd under this symmetry $\left(\theta_{2}=-1\right)$ while all other components of $S_{\mu \nu}$ are even $\left(\theta_{2}=+1\right)$.

Finally, at very high collision energies the source is expected to be approximately invariant under longitudinal boosts within an extended rapidity interval around $Y=0$. If this is the case, the emission function $S(x, \boldsymbol{K})$, when expressed in terms of longitudinal proper time $\tau=\sqrt{t^{2}-z^{2}}$ and space-time rapidity $\eta=\frac{1}{2} \ln [(t+z) /(t-z)]$, depends only on the difference $\eta-Y$ between the space-time and momentumspace rapidities. For equal projectile and target nuclei it then must be an even function of $\eta-Y$, i.e., invariant under a simultaneous sign change of $Y$ and $\eta$. With $z=\tau \sinh \eta$ and $t=\tau \cosh \eta$ this implies

$$
\text { III: } S\left(x, y, z, t ; Y, K_{\perp}, \Phi\right)=S\left(x, y,-z, t ;-Y, K_{\perp}, \Phi\right)
$$

and

$$
\text { III: } S_{\mu \nu}\left(Y, K_{\perp}, \Phi\right)=\theta_{3} S_{\mu \nu}\left(-Y, K_{\perp}, \Phi\right)
$$

with

$$
\theta_{3}=(-1) \delta_{\mu 3}+\delta_{\nu 3}
$$

Combining symmetries II and III allows to relate the spatial correlation tensor at angles $\Phi$ and $\Phi+\pi$ for all rapidities $Y$. For boost-invariant sources, the terms with $\theta_{3}=-1$ (i.e., $S_{03}, S_{13}$, and $S_{23}$ ) vanish at $Y=0$. We note that the symmetry given by Eq. (13) also applies to sources without boostinvariance if they exhibit spatial and momentum anisotropies (i.e., $\Phi$ dependence) already at zero impact parameter, such as fully central collisions (no spectators) between deformed nuclei (e.g., $U+U)$. In this case the source is symmetric under the simultaneous reflection of coordinates and momenta at the transverse plane at $z=0$, in agreement with Eq. (13) [13].

We will concentrate here on the consequences of the combination of symmetries I and II at $Y=0$ and of the combination of all three symmetries at any $Y$. The former case is 
relevant for two-pion correlations at midrapidity in lowenergy collisions between equal spherical nuclei, the latter case applies to high-energy collisions, such as those studied at the heavy-ion colliders RHIC and LHC. Symmetry I alone is less restrictive and is the only useful one when significantly away from midrapidity (in particular in the projectile and target fragmentation regions).

\section{AZIMUTHAL FOURIER DECOMPOSITION OF THE SPATIAL CORRELATION TENSOR}

The above symmetries constrain the $\Phi$ dependence of the components of the spatial correlation tensor [and thereby the implicit $\Phi$ dependence of the HBT radius parameters in Eq. (5)]. Correspondingly, certain expansion coefficients will vanish in an azimuthal Fourier expansion of $S_{\mu \nu}$. Let us write generically $S(\Phi)$ for the $\Phi$ dependence of a given component $S_{\mu \nu}$. Being a real function it has the following Fourier decomposition:

$$
\begin{gathered}
S(\Phi)=\mathcal{C}_{0}+2 \sum_{n=1}^{\infty}\left[\mathcal{C}_{n} \cos (n \Phi)+\mathcal{S}_{n} \sin (n \Phi)\right], \\
\mathcal{C}_{n}=\int_{-\pi}^{\pi} \frac{d \Phi}{2 \pi} S(\Phi) \cos (n \Phi), \\
\mathcal{S}_{n}=\int_{-\pi}^{\pi} \frac{d \Phi}{2 \pi} S(\Phi) \sin (n \Phi) .
\end{gathered}
$$

Symmetry I implies

$$
\begin{aligned}
& \theta_{1}=+1 \Rightarrow \mathcal{S}_{n}=0 \text { for all } n, \\
& \theta_{1}=-1 \Rightarrow \mathcal{C}_{n}=0 \text { for all } n .
\end{aligned}
$$

At $Y=0$, symmetry II eliminates even or odd terms in the Fourier series:

$$
\begin{gathered}
\theta_{2}=+1 \Rightarrow \mathcal{C}_{n}, \mathcal{S}_{n}=0 \text { for odd } n, \\
\theta_{2}=-1 \Rightarrow \mathcal{C}_{n}, \mathcal{S}_{n}=0 \text { for even } n .
\end{gathered}
$$

In fact, Eq. (11) implies a stronger result:

$$
\begin{aligned}
\theta_{2}=+1 \Rightarrow & \mathcal{C}_{n}, \mathcal{S}_{n} \text { are odd (even) functions of } Y \\
& \text { for odd (even) values of } n, \\
\theta_{2}=-1 \Rightarrow & \mathcal{C}_{n}, \mathcal{S}_{n} \text { are odd (even) functions of } Y \\
& \text { for even (odd) values of } n .
\end{aligned}
$$

This will be used in Sec. V. At $Y=0$ Eq. (19) follows from Eq. (20).

Table I lists the Fourier expansions for the components of the spatial correlation tensor at midrapidity that result from

\begin{tabular}{|c|c|c|c|c|}
\hline$S_{\mu \nu}$ & $\theta_{1}$ & $\theta_{2}$ & Fourier expansion & Zeroes \\
\hline$\frac{1}{2}\left\langle\widetilde{x}^{2}+\tilde{y}^{2}\right\rangle$ & 1 & 1 & $A_{0}+2 \Sigma_{n \geqslant 2, \mathrm{even}} A_{n} \cos (n \Phi)$ & \\
\hline$\frac{1}{2}\left\langle\widetilde{x}^{2}-\widetilde{y}^{2}\right\rangle$ & 1 & 1 & $B_{0}+2 \Sigma_{n \geqslant 2, \mathrm{even}} B_{n} \cos (n \Phi)$ & \\
\hline$\langle\tilde{x} \tilde{y}\rangle$ & -1 & 1 & $2 \Sigma_{n \geqslant 2, \mathrm{even}} C_{n} \sin (n \Phi)$ & $0^{\circ}, 90^{\circ}$ \\
\hline$\left\langle\tilde{t}^{2}\right\rangle$ & 1 & 1 & $D_{0}+2 \Sigma_{n \geqslant 2, \mathrm{even}} D_{n} \cos (n \Phi)$ & \\
\hline$\langle\tilde{t} \tilde{x}\rangle$ & 1 & -1 & $2 \Sigma_{n \geqslant 1, \mathrm{odd}} E_{n} \cos (n \Phi)$ & $90^{\circ}$ \\
\hline$\langle\tilde{t} \tilde{y}\rangle$ & -1 & -1 & $2 \Sigma_{n \geqslant 1, \mathrm{odd}} F_{n} \sin (n \Phi)$ & $0^{\circ}$ \\
\hline$\langle\tilde{t} \widetilde{z}\rangle$ & 1 & -1 & $2 \Sigma_{n \geqslant 1, \text { odd }} G_{n} \cos (n \Phi)$ & $90^{\circ}$ \\
\hline$\langle\tilde{x} \tilde{z}\rangle$ & 1 & 1 & $H_{0}+2 \Sigma_{n \geqslant 2, \mathrm{even}} H_{n} \cos (n \Phi)$ & \\
\hline$\langle\tilde{y} \tilde{z}\rangle$ & -1 & 1 & $2 \Sigma_{n \geqslant 2, \mathrm{even}} I_{n} \sin (n \Phi)$ & $0^{\circ}, 90^{\circ}$ \\
\hline$\left\langle\tilde{z}^{2}\right\rangle$ & 1 & 1 & $J_{0}+2 \Sigma_{n \geqslant 2, \mathrm{even}} J_{n} \cos (n \Phi)$ & \\
\hline
\end{tabular}
the combination of these two symmetries. Following Ref. [10] we have used the fact that $S_{11}$ and $S_{22}$ have structurally identical Fourier expansions and combined them into $A$ $=\frac{1}{2}\left(S_{11}+S_{22}\right)$ and $B=\frac{1}{2}\left(S_{11}-S_{22}\right)$, which are the combinations entering in Eqs. (5).
TABLE I. Consequences of symmetries I and II (see text) for the azimuthal Fourier expansion of the spatial correlation tensor $S_{\mu \nu}$ at midrapidity $Y=0$. The last column lists the angles $\Phi$ in the first quadrant where $S_{\mu \nu}\left(Y=0, K_{\perp}, \Phi\right)$ vanishes. The notation follows the one introduced in Ref. [10].

For boost-invariant sources, we can combine symmetries II and III [Eqs. (10) and (13)] to obtain

$$
S_{\mu \nu}\left(Y, K_{\perp}, \Phi\right)=\theta_{2} \theta_{3} S_{\mu \nu}\left(Y, K_{\perp}, \Phi+\pi\right)
$$

For the Fourier coefficients this implies

\begin{tabular}{|c|c|c|c|c|}
\hline$S_{\mu \nu}$ & $\theta_{3}$ & $\theta_{2} \theta_{3}$ & Fourier expansion & Zeroes \\
\hline$\frac{1}{2}\left\langle\widetilde{x}^{2}+\tilde{y}^{2}\right\rangle$ & 1 & 1 & $A_{0}+2 \Sigma_{n \geqslant 2, \mathrm{even}} A_{n} \cos (n \Phi)$ & \\
\hline$\frac{1}{2}\left\langle\widetilde{x}^{2}-\widetilde{y}^{2}\right\rangle$ & 1 & 1 & $B_{0}+2 \Sigma_{n \geqslant 2, \mathrm{even}} B_{n} \cos (n \Phi)$ & \\
\hline$\langle\tilde{x} \tilde{y}\rangle$ & 1 & 1 & $2 \Sigma_{n \geqslant 2, \text { even }} C_{n} \sin (n \Phi)$ & $\Phi=0^{\circ}, 90^{\circ}$ \\
\hline$\left\langle\widetilde{t}^{2}\right\rangle$ & 1 & 1 & $D_{0}+2 \Sigma_{n \geqslant 2, \mathrm{even}} D_{n} \cos (n \Phi)$ & \\
\hline$\langle\tilde{t} \tilde{x}\rangle$ & 1 & -1 & $2 \Sigma_{n \geqslant 1, \mathrm{odd}} E_{n} \cos (n \Phi)$ & $\Phi=90^{\circ}$ \\
\hline$\langle\tilde{t} \tilde{y}\rangle$ & 1 & -1 & $2 \sum_{n \geqslant 1, \text { odd }} F_{n} \sin (n \Phi)$ & $\Phi=0^{\circ}$ \\
\hline$\langle\tilde{t} \widetilde{z}\rangle$ & -1 & 1 & $G_{0}+2 \Sigma_{n \geqslant 2, \mathrm{even}} G_{n} \cos (n \Phi)$ & $Y=0$ \\
\hline$\langle\tilde{x} \tilde{z}\rangle$ & -1 & -1 & $2 \Sigma_{n \geqslant 1, \mathrm{odd}} H_{n} \cos (n \Phi)$ & $Y=0, \Phi=90^{\circ}$ \\
\hline$\langle\tilde{y} \widetilde{z}\rangle$ & -1 & -1 & $2 \Sigma_{n \geqslant 1, \text { odd }} I_{n} \sin (n \Phi)$ & $Y=0, \Phi=0^{\circ}$ \\
\hline$\left\langle\tilde{z}^{2}\right\rangle$ & 1 & 1 & $J_{0}+2 \Sigma_{n \geqslant 2, \text { even }} J_{n} \cos (n \Phi)$ & \\
\hline
\end{tabular}

$$
\begin{aligned}
& \theta_{2} \theta_{3}=+1 \Rightarrow \mathcal{C}_{n}, \mathcal{S}_{n}=0 \text { for odd } n, \\
& \theta_{2} \theta_{3}=-1 \Rightarrow \mathcal{C}_{n}, \mathcal{S}_{n}=0 \text { for even } n
\end{aligned}
$$

In contrast to Eqs. (19) this is now true for all rapidities $Y$. The corresponding Fourier expansions are listed in Table II. Note that according to Eq. (20) the nonvanishing coefficients $G_{n}, H_{n}$, and $I_{n}$ in Table II are odd functions of rapidity and thus vanish at $Y=0$.

TABLE II. Consequences of symmetries I, II, and III (see text) for the azimuthal Fourier expansion of the spatial correlation tensor $S_{\mu \nu}$ for boost-invariant sources. The last column lists the rapidities $Y$ and angles $\Phi$ in the first quadrant, where $S_{\mu \nu}\left(Y, K_{\perp}, \Phi\right)$ vanishes. 


\section{FOURIER EXPANSION OF THE RADIUS PARAMETERS}

We will now combine the above implicit $\Phi$ dependence of the spatial correlation tensor with the explicit $\Phi$ dependence shown in Eqs. (5). When studying the combination of symmetries I and II for sources which are not invariant under longitudinal boosts, we must restrict our attention to midrapidity pairs with $\beta_{l}=0$. For simplicity, we also set $\beta_{l}=0$ in the boost-invariant case; this means that we are studying the correlation radii in the longitudinally comoving system (LCMS) [1]. General expressions for $\beta_{l} \neq 0$ are easily obtained by boosting from the LCMS to a fixed longitudinal reference frame and can be found in Refs. $[14,15]$. For boostinvariant sources $R_{s l}^{2}=0$ independent of rapidity $[14,15]$; without boost invariance this is generally not even true at midrapidity [see Eqs. (5) and Table I]. As shown in Ref. [11], a nonzero value for $R_{s l}^{2}$ arises naturally if the longitudinal major axis of the source ellipsoid is tilted away from the beam direction; longitudinal boost invariance forbids such a tilt.

Using the symbols introduced in Tables I and II and setting $\beta_{l}=0$, Eqs. (5) simplify to

$$
\begin{aligned}
R_{s}^{2}= & A-B \cos (2 \Phi)-C \sin (2 \Phi), \\
R_{o}^{2}= & A+B \cos (2 \Phi)+C \sin (2 \Phi) \\
& -2 \beta_{\perp}(E \cos \Phi+F \sin \Phi)+\beta_{\perp}^{2} D \\
R_{o s}^{2}= & C \cos (2 \Phi)-B \sin (2 \Phi)+\beta_{\perp}(E \sin \Phi-F \cos \Phi), \\
R_{l}^{2}= & J \\
R_{o l}^{2}= & H \cos \Phi+I \sin \Phi-\beta_{\perp} G \\
R_{s l}^{2}= & I \cos \Phi-H \sin \Phi .
\end{aligned}
$$

Here $A, B, \ldots, J$ are functions of $\Phi$, whose Fourier expansions are given in Tables I and II. For a boost-invariant source $G(\Phi), H(\Phi)$ and $I(\Phi)$ (i.e., $R_{o l}^{2}$ and $R_{s l}^{2}$ ) vanish at $\beta_{l}=Y=0$. A comparison of Tables I and II shows that at $Y$ $=0$ all other $S_{\mu \nu}$ components entering Eqs. (23) have exactly the same Fourier expansion with and without longitudinal boost invariance. We may, therefore, investigate Eqs. (23) on the basis of the expansions listed in Table I and recover the boost-invariant case later by simply setting $R_{o l}$ $=R_{s l}=0$.

In Ref. [11] we studied the limit of vanishing implicit $\Phi$ dependence (i.e., $S_{\mu \nu}$ does not depend on $\Phi$ ). Table I shows that in this limit only the diagonal elements and $S_{13}$ (i.e., $A, B, D, H$, and $J$ ) are nonzero. As discussed in Ref. [11], this limit requires only that space-momentum correlations (e.g., due to collective flow effects) are weak. A more general situation was analyzed in Ref. [10], where terms up to $n=2$ were kept in the Fourier expansion of the HBT radii, but in that work the $\Phi$ dependences of the emission duration and space-time correlations were neglected relative to those of the spatial components $S_{i j}$. We here remove both of these approximations.

Inserting the expansions in Table I into Eq. (23) and using

$$
\begin{aligned}
& 2 \cos n \Phi \cos m \Phi=\cos (n-m) \Phi+\cos (n+m) \Phi, \\
& 2 \sin n \Phi \sin m \Phi=\cos (n-m) \Phi-\cos (n+m) \Phi, \\
& 2 \cos n \Phi \sin m \Phi=\sin (n+m) \Phi-\sin (n-m) \Phi,
\end{aligned}
$$

we see that at midrapidity the HBT radius parameters $R_{\alpha}^{2}\left(Y=0, K_{\perp}, \Phi\right)$ have the following Fourier expansions:

$$
\begin{aligned}
& R_{s}^{2}=R_{s, 0}^{2}+2 \sum_{n=2,4,6, \ldots} R_{s, n}^{2} \cos (n \Phi), \\
& R_{o}^{2}=R_{o, 0}^{2}+2 \sum_{n=2,4,6, \ldots} R_{o, n}^{2} \cos (n \Phi),
\end{aligned}
$$$$
R_{o s}^{2}=\quad 2 \sum_{n=2,4,6, \ldots} R_{o s, n}^{2} \sin (n \Phi),
$$$$
R_{l}^{2}=R_{l, 0}^{2}+2 \sum_{n=2,4,6, \ldots} R_{l, n}^{2} \cos (n \Phi),
$$$$
R_{o l}^{2}=\quad 2 \sum_{n=1,3,5, \ldots} R_{o l, n}^{2} \cos (n \Phi)
$$$$
R_{s l}^{2}=\quad 2 \sum_{n=1,3,5, \ldots} R_{s l, n}^{2} \sin (n \Phi) .
$$

Due to the symmetries of the emission function, the HBT radius parameters are sums of either cosine or sine terms, involving either even or odd multiples of the emission angle $\Phi$, but no mixtures of different such terms. As a consequence, $R_{o s}^{2}$ vanishes at both $\Phi=0^{\circ}$ and $90^{\circ}$ (i.e., its leading contribution features a second order harmonic oscillation as a function of the emission angle). $R_{o l}^{2}$ and $R_{s l}^{2}$ in general exhibit leading first-order harmonic oscillations [11] with zeroes at $90^{\circ}$ and $0^{\circ}$, respectively. For a boost invariant source they vanish identically.

The Fourier coefficients $R_{\alpha, n}^{2}$ are functions of $K_{\perp}$. We now list them up to order $n=2$. The $\Phi$-independent terms are given by

$$
\begin{aligned}
& R_{s, 0}^{2}=A_{0}-B_{2}-C_{2}, \\
& R_{o, 0}^{2}=A_{0}+B_{2}+C_{2}-2 \beta_{\perp}\left(E_{1}+F_{1}\right)+\beta_{\perp}^{2} D_{0}, \\
& R_{l, 0}^{2}=J_{0} .
\end{aligned}
$$

The coefficients of the first-order harmonics are 


$$
\begin{aligned}
& R_{o l, 1}^{2}=\frac{1}{2}\left(H_{0}+H_{2}+I_{2}\right)-\beta_{\perp} G_{1}, \\
& R_{s l, 1}^{2}=\frac{1}{2}\left(-H_{0}+H_{2}+I_{2}\right) .
\end{aligned}
$$

For a boost-invariant source these vanish. The term $\sim H_{0}$ describes the tilt of the emission region relative to the beam axis that was discussed in Ref. [11]. The second-order harmonic oscillations have amplitudes

$$
\begin{aligned}
R_{s, 2}^{2}= & A_{2}-\frac{1}{2}\left(B_{0}+B_{4}+C_{4}\right), \\
R_{o, 2}^{2}= & A_{2}+\frac{1}{2}\left(B_{0}+B_{4}+C_{4}\right) \\
& -\beta_{\perp}\left(E_{1}+E_{3}-F_{1}+F_{3}\right)+\beta_{\perp}^{2} D_{2}, \\
R_{o s, 2}^{2}= & \frac{1}{2}\left(-B_{0}+B_{4}+C_{4}\right)+\frac{\beta_{\perp}}{2}\left(E_{1}-E_{3}-F_{1}-F_{3}\right), \\
R_{l, 2}^{2}= & J_{2} .
\end{aligned}
$$

If the emission duration $D=\left\langle\widetilde{t}^{2}\right\rangle$ is independent of emission angle $\left(D_{2,4,6, \ldots} \approx 0\right)$ and all higher-order harmonics $n \geqslant 3$ of the spatial correlation tensor are small, these amplitudes fulfill the approximate "sum rule" [10]

$$
\begin{aligned}
R_{o, 2}^{2} & -R_{s, 2}^{2}+2 R_{o s, 2}^{2} \\
& =2\left(B_{4}+C_{4}\right)-2 \beta_{\perp}\left(E_{3}+F_{3}\right)+\beta_{\perp}^{2} D_{2} \\
& \approx 0 .
\end{aligned}
$$

Note that the leading first-order harmonics of $\langle\tilde{t} \tilde{x}\rangle$ and $\langle\tilde{t} \tilde{y}\rangle$, which describe how the transverse positions are correlated with time at freeze-out, cancel in this "sum rule." If the data satisfy this "sum rule" for all values of $K_{\perp}$ resp. $\beta_{\perp}$, one may conclude (barring unlikely accidental cancellations among the terms) that $D_{2}, E_{3}, F_{3}, B_{4}$, and $C_{4}$ all vanish. In this case the azimuthal oscillation amplitudes of the transverse HBT radii reduce to

$$
\begin{aligned}
& R_{s, 2}^{2}=A_{2}-\frac{1}{2} B_{0}, \\
& R_{o, 2}^{2}=A_{2}+\frac{1}{2} B_{0}-\beta_{\perp}\left(E_{1}-F_{1}\right), \\
& R_{o s, 2}^{2}=-\frac{1}{2} B_{0}+\frac{1}{2} \beta_{\perp}\left(E_{1}-F_{1}\right) .
\end{aligned}
$$

The term $\sim\left(E_{1}-F_{1}\right)$ is the leading (first harmonic) contribution to the correlation $\langle(\tilde{x}-\tilde{y}) \tilde{t}\rangle$ between emission points and times. In a hydrodynamic model this term reflects the geometric manifestation of elliptic flow, namely, that the freezeout radius increases with time more rapidly in $x$ than in $y$ direction [16]. Since it comes with an explicit factor of $\beta_{\perp}$, one may be able to isolate it using the $K_{\perp}$ dependence of the azimuthal oscillation amplitudes [see Eqs. (30)] at small $K_{\perp}$.

Finally, in the absence of dynamical space-momentum correlations, all implicit $\Phi$ dependences (i.e., all higher harmonics in Table I) are expected to vanish, leading to the "geometric relations" [10]

$$
\begin{aligned}
& R_{s, 0}^{2}=A_{0}, \\
& R_{o, 0}^{2}-R_{s, 0}^{2}=\beta_{\perp}^{2} D_{0}, \\
& R_{l, 0}^{2}=J_{0}, \\
& R_{o l, 1}^{2}=-R_{s l, 1}^{2}=\frac{1}{2} H_{0}, \\
& R_{o, 2}^{2}=-R_{s, 2}^{2}=-R_{o s, 2}^{2}=\frac{1}{2} B_{0} .
\end{aligned}
$$

In this case all five nonvanishing components of the spatial correlation tensor can be separated [11].

\section{CONSIDERATIONS FOR A FINITE SYMMETRIC WINDOW AROUND $Y=0$}

The results quoted so far were derived at midrapidity $Y$ $=0$ since, at least in the absence of longitudinal boost invariance, symmetry II can only there be used to constrain the azimuthal Fourier expansion of $S_{\mu \nu}$, by eliminating either even or odd terms in the sums over $n$ [see Eq. (19)]. In practice, statistical limitations render strict cuts on the pair rapidity $Y$ quite painful. It is, therefore, important to assess the necessary modifications if the data are collected in a finite size rapidity interval around $Y=0$. We now prove the important result that, as long as the HBT radii are obtained from averaging over a symmetric rapidity interval around $Y$ $=0$, the general form [see Eqs. (25)] of their Fourier expansions remains unchanged. On the other hand, Eqs. (26)-(28) receive additional contributions which, at leading order in the width $\Delta Y$ of the rapidity interval, grow quadratically as $(\Delta Y)^{2}$; this can be used to eliminate them by varying $\Delta Y$ and extrapolating to $\Delta Y=0$.

As noted in Eq. (20), the point reflection symmetry [see Eqs. (10) and (11)] allows to classify the Fourier expansion coefficients of the spatial correlation tensor $S_{\mu \nu}$ as either even or odd functions of rapidity $Y$. The odd terms vanish at $Y=0$, but do not do so any longer at $Y \neq 0$. However, when calculating the HBT radii from $S_{\mu \nu}$ according to Eqs. (5) and averaging them over a finite, but symmetric rapidity interval around $Y=0$, terms which are odd in $Y$ average to zero. Therefore, there are no new contributions to $R_{s}^{2}, R_{o}^{2}$ and $R_{o s}^{2}$ in this case. $R_{l}^{2}, R_{o l}^{2}$ and $R_{s l}^{2}$, on the other hand, contain at $Y \neq 0$ additional terms beyond those listed in Eqs. (23) that are multiplied by either one or two powers of $\beta_{l}$. When multiplying an odd Fourier coefficient by $\beta_{l}$, the result is even in $\beta_{l}$ (respectively, $Y$ ) and does not average to zero across the rapidity interval $\Delta Y$. In fact, at leading order in 
$\Delta Y$, its average is $\sim\left\langle\beta_{l}^{2}\right\rangle$ which grows quadratically with $\Delta Y$.

Let us now look at how these extra terms modify the Fourier expansions given in the last three lines of Eq. (25). We begin with $R_{l}^{2}=S_{33}-2 \beta_{l} S_{03}+\beta_{l}^{2} S_{00}$ and average it over the symmetric interval $\Delta Y$. Table I tells us that the Fourier coefficients of $S_{33}$ with odd values of $n$ are odd functions of $Y$ and thus average to zero. For $S_{03}$ the coefficients with odd $n$ are even functions of $Y$, but since $S_{03}$ is multiplied by $\beta_{l}$, these odd $n$ terms again average to zero. The same is true for the last term, where the factor $\beta_{l}^{2}$ preserves the $Y$-reflection symmetries of the expansion coefficients. Altogether, the rapidity-averaged longitudinal radius $\left\langle R_{l}^{2}\right\rangle$ continues to have only even $n$ terms in its Fourier expansion, just as Eq. (25) states for $Y=0$. In the same fashion one also shows that the rapidity-averaged radius parameters $\left\langle R_{o l}^{2}\right\rangle$ and $\left\langle R_{s l}^{2}\right\rangle$ continue to have the same Fourier expansions as in Eq. (25). In other words, averaging the HBT radii over a finite, symmetric rapidity interval around $Y=0$ preserves the general structure [given by Eq. (25)] of their azimuthal Fourier expansions.

When expressing the Fourier components of the rapidityaveraged HBT radii in terms of the harmonic coefficients of $S_{\mu \nu}$, new terms arise, and Eqs. (26)-(28) are modified. We only list those equations whose structure changes:

$$
\begin{aligned}
\left\langle R_{l, 0}^{2}\right\rangle= & \left\langle J_{0}\right\rangle-2\left\langle\beta_{l} G_{0}\right\rangle+\left\langle\beta_{l}^{2} D_{0}\right\rangle, \\
\left\langle R_{l, 2}^{2}\right\rangle= & \left\langle J_{2}\right\rangle-2\left\langle\beta_{l} G_{2}\right\rangle+\left\langle\beta_{l}^{2} D_{2}\right\rangle, \\
\left\langle R_{o l, 1}^{2}\right\rangle= & \frac{1}{2}\left\langle H_{0}+H_{2}+I_{2}-\beta_{l}\left(E_{0}+E_{2}+F_{2}\right)\right\rangle \\
& -\beta_{\perp}\left\langle G_{1}-\beta_{l} D_{1}\right\rangle, \\
\left\langle R_{s l, 1}^{2}\right\rangle= & \frac{1}{2}\left\langle-H_{0}+H_{2}+I_{2}-\beta_{l}\left(-E_{0}+E_{2}+F_{2}\right)\right\rangle .
\end{aligned}
$$

Here the angular brackets denote the average over the symmetric rapidity interval $\Delta Y$. All terms involving one or two explicit factors $\beta_{l}$ vanish quadratically as $\Delta Y \rightarrow 0$ in which limit Eqs. (26)-(28) are recovered.

\section{WHAT IF THE SIGN OF THE IMPACT PARAMETER CANNOT BE DETERMINED?}

If the orientation of the reaction plane is reconstructed from an even Fourier component of the single-particle distribution (e.g., from the elliptic flow coefficient $v_{2}$ as is the case at RHIC), the direction of the impact parameter vector $\boldsymbol{b}$ has a sign ambiguity, i.e., after aligning events according to their reaction plane the event sample contains equal contributions from collisions with impact parameters $\boldsymbol{b}$ and $\boldsymbol{- b}$. This ambiguity does not exist if the event plane is reconstructed from the directed flow coefficient $v_{1}$ (as one does at the AGS and SPS) whose sign has a one-to-one correlation with the direction of $\boldsymbol{b}$ within the reaction plane.

If events with impact parameters $\boldsymbol{b}$ and $-\boldsymbol{b}$ are equally mixed, the effective source function is symmetric under the exchange $\boldsymbol{b} \rightarrow-\boldsymbol{b}$ which is equivalent to an azimuthal rotation by $180^{\circ}$ :

$$
\text { IIa: } S\left(x, y, z, t ; Y, K_{\perp}, \Phi\right)=S\left(-x,-y, z, t ; Y, K_{\perp}, \Phi+\pi\right) \text {. }
$$

For the spatial correlation tensor this implies

$$
\text { IIa: } S_{\mu \nu}\left(Y, K_{\perp}, \Phi\right)=\theta_{2 a} S_{\mu \nu}\left(Y, K_{\perp}, \Phi+\pi\right)
$$

with

$$
\theta_{2}=(-1)^{\delta_{\mu 1}+\delta_{\nu 1}+\delta_{\mu 2}+\delta_{\nu 2}}
$$

One easily checks that the $\operatorname{sign} \theta_{2 a}$ is, in fact, equal to the product $\theta_{2} \theta_{3}$ of the signs under symmetries II and III, as tabulated in Table II. Correspondingly, the general form of the Fourier expansions of $S_{\mu \nu}$ and of the HBT radii are exactly the same as those listed in and resulting from Table II for a longitudinally boost-invariant source. We see in particular that a sign ambiguity for the direction of the impact parameter automatically leads to vanishing cross terms $R_{o l}^{2}$ and $R_{s l}^{2}$.

\section{CORRECTIONS FOR BINNING AND FINITE EVENT-PLANE RESOLUTION}

Experimentally, the two-pion correlation function is obtained as the ratio of correlated (true) pairs, $N(\boldsymbol{q}, \boldsymbol{K})$, and uncorrelated (mixed event) pairs, $D(\boldsymbol{q}, \boldsymbol{K})$. In an azimuthally sensitive analysis, one constructs these distributions for a given selection on emission angle $\Phi$. However, finite binning in $\Phi$ and uncertainty in the experimental estimation of the reaction plane tend to dampen the azimuthal dependencies of the observed ("raw") distributions $N_{\exp }(\boldsymbol{q}, \boldsymbol{K})$ and $D_{\exp }(\boldsymbol{q}, \boldsymbol{K})$. In this section we present a model-independent procedure to correct for these effects.

Since the reaction plane is reconstructed event-by-event from the anisotropies of the single-particle momentum distribution [2-4], its orientation is only known with a finite statistical accuracy controlled by the magnitude of the anisotropies and the number of particles used in the reconstruction process. Correspondingly, in a statistical average over the event sample the true reaction plane angle $\psi_{R}$ is distributed around the reconstructed one $\psi_{m}$ by a probability distribution [see Eq. (9) in Ref. [4]]

$$
\begin{aligned}
& p\left(\psi_{m}-\psi_{R}\right) \equiv \frac{d P}{d\left(m\left(\psi_{m}-\psi_{R}\right)\right)} \\
& =\int \frac{v_{m}^{\prime} d v_{m}^{\prime}}{2 \pi \sigma^{2}} \exp \left[-\frac{v_{m}^{2}+v_{m}^{\prime 2}-2 v_{m} v_{m}^{\prime} \cos \left(m\left(\psi_{m}-\psi_{R}\right)\right)}{2 \sigma^{2}}\right],
\end{aligned}
$$


with width $\sigma^{2}=\left\langle w^{2}\right\rangle /\left(2 M\langle w\rangle^{2}\right)$ [where, $M$ is the number of particles per event and $w$ is an arbitrary weight function (e.g., $w=1$ or $w=p_{\perp}$ ) used in the analysis]. $m$ denotes the order of the Fourier component of the single-particle spectrum used to extract the reaction plane, and $v_{m}$ is the corresponding Fourier coefficient; the cases $m=1$ (directed flow) and $m=2$ (elliptic flow) are relevant in practice. Correspondingly, a measurement of the two-particle distributions $N(\boldsymbol{q}, \boldsymbol{K})$ and $D(\boldsymbol{q}, \boldsymbol{K})$ at fixed emission angle $\Phi \equiv \Phi-\psi_{m}$ relative to the reconstructed event-plane corresponds to an average of the real two-particle distributions over a range of emission angles $\Phi-\psi_{R}$ relative to the true reaction plane, where the average is taken with the distribution of Eq. (36). The averaging reduces the azimuthal dependence of the correlation function (and of the HBT radii extracted from it) and must be corrected for, before comparing to models.

An additional smearing which goes in the same direction arises from the binning of the data in $\Phi$. By summing the data over all emission angles $\phi$ within an interval of width $\Delta$ centered at $\Phi$, one effectively performs an additional smearing of $N(\boldsymbol{q}, \boldsymbol{K})$ and $D(\boldsymbol{q}, \boldsymbol{K})$ over the azimuthal emission angle with the distribution

$$
f_{\Delta}(\phi-\Phi)=\frac{1}{\Delta} \theta\left(\phi-\Phi+\frac{1}{2} \Delta\right) \theta\left(\frac{1}{2} \Delta-\phi+\Phi\right) .
$$

The two effects can be combined by folding the distributions $p$ and $f_{\Delta}$ and averaging the true correlated and mixed pair distributions with

$$
H_{\Delta}(\phi)=\int_{-\pi}^{\pi} d \psi p(\psi) f_{\Delta}(\phi-\psi)=\frac{1}{\Delta} \int_{-\Delta / 2}^{\Delta / 2} d \theta p(\phi-\theta) .
$$

The above azimuthal averaging affects the numerator $N$ and denominator $D$ separately. Suppressing the dependence on $K_{\perp}$ and $Y$ for clarity, the measured angular dependence of the correlated pairs relative to the reconstructed reaction plane $\psi_{m}, N_{\text {exp }}\left(\boldsymbol{q}, \Phi-\psi_{m}\right)$, is related to their true angular dependence relative to the real reaction plane $\psi_{R}, N(\boldsymbol{q}, \Phi$ $\left.-\psi_{R}\right)$, by

$$
\begin{aligned}
N_{\exp }\left(\boldsymbol{q}, \Phi-\psi_{m}\right)= & \int_{-\pi}^{\pi} d \boldsymbol{\phi} N_{\Delta}\left(\boldsymbol{q}, \boldsymbol{\phi}-\psi_{R}\right) \\
& \times p\left(\left(\Phi-\psi_{m}\right)-\left(\phi-\psi_{R}\right)\right),
\end{aligned}
$$

where

$$
N_{\Delta}\left(\boldsymbol{q}, \phi-\psi_{R}\right)=\int_{\phi-\psi_{R}-\Delta / 2}^{\phi-\psi_{R}+\Delta / 2} N(\boldsymbol{q}, \theta) d \theta
$$

denotes the effect of summing the data in angular bins of width $\Delta$. An analogous pair of equations holds for the measured and true uncorrelated pairs in the denominator, $D_{\exp }\left(\boldsymbol{q}, \Phi-\psi_{m}\right)$ and $D\left(\boldsymbol{q}, \boldsymbol{\phi}-\psi_{R}\right)$.

The task at hand is to extract the true angular dependence on $\Phi-\psi_{R}$ from the measured dependence on $\Phi_{j}-\psi_{m}$, where $j$ labels the angular bins centered at angles $\Phi_{j}$ relative to the reconstructed reaction plane. To this end we Fourier decompose the measured quantities $N$ and $D$ for each value of $\boldsymbol{q}$. For example

$$
\begin{aligned}
N_{\exp }\left(\boldsymbol{q}, \Phi-\psi_{m}\right)= & N_{0}^{\exp }(\boldsymbol{q})+2 \sum_{n=1}^{n_{\text {bin }}}\left[N_{c, n}^{\exp }(\boldsymbol{q}) \cos \left(n\left(\Phi-\psi_{m}\right)\right)\right. \\
& \left.+N_{s, n}^{\exp }(\boldsymbol{q}) \sin \left(n\left(\Phi-\psi_{m}\right)\right)\right], \\
N_{c, n}^{\exp }(\boldsymbol{q}) \equiv\left\langle N_{\exp }(\boldsymbol{q}, \Phi) \cos (n \Phi)\right\rangle & \\
= & \frac{1}{n_{\text {bin }}} \sum_{j=1}^{n_{\text {bin }}} N_{\exp }\left(\boldsymbol{q}, \Phi_{j}\right) \cos \left(n \Phi_{j}\right), \\
N_{s, n}^{\exp }(\boldsymbol{q}) & \equiv\left\langle N_{\exp }(\boldsymbol{q}, \Phi) \sin (n \Phi)\right\rangle \\
= & \frac{1}{n_{\text {bin }}} \sum_{j=1}^{n_{\text {bin }}} N_{\exp }\left(\boldsymbol{q}, \Phi_{j}\right) \sin \left(n \Phi_{j}\right),
\end{aligned}
$$

where $n_{\text {bin }}$ denotes the number of angular bins (for finite $n_{\text {bin }}$ only Fourier components with $n \leqslant n_{\text {bin }}$ are meaningful). We further imagine doing the same for the corresponding true and binned quantities corrected for event-plane resolution:

$$
\begin{gathered}
N\left(\boldsymbol{q}, \Phi-\psi_{R}\right)=N_{0}(\boldsymbol{q})+2 \sum_{n=1}^{n_{\text {bin }}}\left[N_{c, n}(\boldsymbol{q}) \cos \left(n\left(\Phi-\psi_{R}\right)\right)\right. \\
\left.+N_{s, n}(\boldsymbol{q}) \sin \left(n\left(\Phi-\psi_{R}\right)\right)\right], \\
N_{\Delta}(\boldsymbol{q}, \theta)=N_{0}^{\Delta}(\boldsymbol{q})+2 \sum_{n=1}^{n_{\text {bin }}}\left[N_{c, n}^{\Delta}(\boldsymbol{q}) \cos (n \theta)\right. \\
\left.+N_{s, n}^{\Delta}(\boldsymbol{q}) \sin (n \theta)\right] .
\end{gathered}
$$

Analogous expressions hold for the mixed pairs in the denominator $D$. Inserting the Fourier expansions of Eq. (42) into Eqs. (39) and (40), using that the distributions $p$ and $f_{\Delta}$ are even functions of their arguments, and comparing the result with Eq. (41) one easily finds for all $n$ and both series of coefficients ( $\alpha=c$ or $s$ ):

$$
\begin{aligned}
& N_{\alpha, n}^{\Delta}(\boldsymbol{q})=N_{\alpha, n}(\boldsymbol{q}) \frac{\sin (n \Delta / 2)}{n \Delta / 2}, \\
& N_{\alpha, n}^{\exp }(\boldsymbol{q})=N_{\alpha, n}^{\Delta}(\boldsymbol{q})\left\langle\cos \left(n\left(\psi_{m}-\psi_{R}\right)\right)\right\rangle_{p} .
\end{aligned}
$$

The factors $\left\langle\cos \left(n\left(\psi_{m}-\psi_{R}\right)\right)\right\rangle_{p}$, arising from an average over the event-plane distribution [see Eq. (36)], are the wellknown correction factors for event-plane resolution arising in the process of extracting the anisotropic flow coefficients $v_{n}$ from the single-particle spectrum [2-4].

Given Eq. (43), the numerator $N$ and denominator $D$ of the correlation function at each measured angle $\Phi_{j}$ and relative momentum $\boldsymbol{q}$ can now be easily corrected for the effects of angular binning and finite event-plane resolution (setting again $\psi_{m}=0$ ): 


$$
\begin{aligned}
N\left(\boldsymbol{q}, \Phi_{j}\right)= & N_{\exp }\left(\boldsymbol{q}, \Phi_{j}\right)+2 \sum_{n=1}^{n_{\text {bin }}} \zeta_{n, m}(\Delta)\left[N_{c, n}^{\exp }(\boldsymbol{q}) \cos \left(n \Phi_{j}\right)\right. \\
& \left.+N_{s, n}^{\exp }(\boldsymbol{q}) \sin \left(n \Phi_{j}\right)\right]
\end{aligned}
$$

with correction parameters $\zeta_{n, m}(\Delta)$ given by the simple expression

$$
\zeta_{n, m}(\Delta)=\frac{n \Delta / 2}{\sin (n \Delta / 2)\left\langle\cos \left(n\left(\psi_{m}-\psi_{R}\right)\right)\right\rangle_{p}}-1
$$

A similar equation holds for the uncorrelated pairs in the denominator $D$. Since the right-hand side of Eq. (44) involves only experimentally known quantities, the correction algorithm is model independent. The sums over $n$ go over all allowed values; if $m$ is even (i.e., the sign of the impact parameter is not known), both $N$ and $D$ are symmetric under azimuthal rotations by $180^{\circ}$ and only even values of $n$ are summed over. Contrary to the HBT radius parameters or to single-particle flow measures [4], $N$ and $D$ have no unique symmetry under $\Phi \rightarrow-\Phi$; thus in general both sine and cosine terms contribute to the sum in Eq. (44).

After applying the correction given by Eq. (44) to the data, the ratio $C(\boldsymbol{q}, \boldsymbol{K})=N(\boldsymbol{q}, \boldsymbol{K}) / D(\boldsymbol{q}, \boldsymbol{K})$ gives the corrected two-particle correlation function from which all angular binning and event-plane resultion effects have been removed. The true emission angle dependence of the HBT radius parameters can thus be directly extracted from a Gaussian fit with Eq. (3) to this function $C(\boldsymbol{q}, \boldsymbol{K})$.

\section{SUMMARY}

Equations (25) give the most general Fourier expansions for the HBT radius parameters at midrapidity which are con- sistent with the symmetries of the source in noncentral collisions between equal mass spherical nuclei. For full-overlap central collisions between deformed nuclei (e.g., $U+U$ ) and for longitudinally boost-invariant sources they also apply at $Y \neq 0$. The structure of these expansions is preserved if the data are averaged over a symmetric finite rapidity interval around $Y=0$. They provide a basis for fitting the azimuthal emission angle dependence of experimentally determined correlation functions. A model-independent correction of the measured two-pion correlation function for event-plane resolution and angular binning effects is possible and given in Sec. VII. Equations (26)-(28), (31), and (32) relate the oscillation amplitudes extracted from the thus corrected correlation function to the leading harmonic coefficients of the spatial correlation tensor and allow to constrain models for the emission function using azimuthally sensitive HBT data. If the emission duration is independent of emission angle and high-order harmonics of the spatial correlation tensor are small, then spatial and temporal aspects of the emission function can be separated.

\section{ACKNOWLEDGMENTS}

We gratefully acknowledge fruitful discussions with $\mathrm{P}$. Kolb and B. Tomásik. This work was supported in part by the U.S. Department of Energy under Contract No. DEFG02-01ER41190 and by the National Science Foundation under Grant Nos. PHY99-07949 and PHY00-99476. U.H. acknowledges the warm hospitality of the Kavli Institute for Theoretical Physics at the University of Santa Barbara during the Workshop Program "QCD in the RHIC Era."
[1] U.A. Wiedemann and U. Heinz, Phys. Rep. 319, 145 (1999); U. Heinz and B.V. Jacak, Annu. Rev. Nucl. Part. Sci. 49, 529 (1999).

[2] J.Y. Ollitrault, Phys. Rev. D 48, 1132 (1993).

[3] S. Voloshin and Y. Zhang, Z. Phys. C 70, 665 (1996).

[4] A.M. Poskanzer and S.A. Voloshin, Phys. Rev. C 58, 1671 (1998).

[5] H. Stöcker and W. Greiner, Phys. Rep. 137, 277 (1986).

[6] J.Y. Ollitrault, Phys. Rev. D 46, 229 (1992).

[7] E895 Collaboration, M.A. Lisa et al., Phys. Lett. B 496, 1 (2000).

[8] STAR Collaboration, F. Retiǵere et al., nucl-ex/0111013.

[9] S.A. Voloshin and W.E. Cleland, Phys. Rev. C 53, 896 (1996);
54, 3212 (1996).

[10] U.A. Wiedemann, Phys. Rev. C 57, 266 (1998).

[11] M.A. Lisa, U. Heinz, and U.A. Wiedemann, Phys. Lett. B 489, 287 (2000).

[12] U. Heinz and P.F. Kolb, Nucl. Phys. A702, 269 (2002); hep-ph/0204061.

[13] We thank Peter Kolb for this remark.

[14] S. Chapman, P. Scotto, and U. Heinz, Heavy Ion Phys. 1, 1 (1995).

[15] S. Chapman, J.R. Nix, and U. Heinz, Phys. Rev. C 52, 2694 (1995).

[16] U. Heinz and P. F. Kolb, Phys. Lett. B 542, 216 (2002). 